\title{
INTERPRETATION OF SOME PROPERTIES OF \\ EXTRAGALACTIC JETS IN THE CONTEXT OF A TWO \\ COMPONENT MODEL
}

\author{
LOURDES VICENTE \\ Observatoire de Paris-Meudon, DARC, France
}

We have studied a sample of extragalactic jets within the context of a two component model. This model supposes the existence of two flows in the extragalactic jets. An electron-positron fast beam coming from the internal regions of the accretion disk, responsible for the VLBI jet at the parsec scale and for the observed superluminal speeds. A second slow component, responsible for the jet observed at larger scales. The fast beam is destroyed when the parallel magnetic field is smaller than a critical value, $B_{I I}<B_{\text {crit }}=3.2 \times 10^{-3} n_{p}^{1 / 2}$, where $n_{p}$ is the density of the background thermal plasma of the slow component.

The present sample includes 9 FRIs, 12 WATs, 1 FRII, 1 CSS and 1 Core-Halo. The critical zone was determined by careful examination of published radio maps looking for regions along the jets where there was an important morphological change, like bright knots, gaps or strong sudden polarization changes.

The identification of the critical zone fixes the internal density $n_{p}$ from the magnetic field, deduced from standard equipartition argument. This allows us to estimate the mass outflows and energy flux for the slow and fast components, $\dot{M}_{0}=S_{0} n_{p} m_{e} m_{e} V_{0}, \dot{M}_{f}=S_{f} \gamma n_{f} m_{e} c, K_{0}=S_{0} V_{0}\left(n_{p} m_{p} V_{0}^{2} / 2+B^{2} / 6+5 n_{p} K T / 2\right)$ and $K_{f}=S_{f} \gamma^{2}(\gamma-1) n_{f} m_{e} c^{3}$. Cross sectional areas $S_{0}$ and $S_{f}$ are deduced from the radio maps. We assumed $\gamma=4$ and $T=10^{8} \mathrm{~K}$. We have taken the density of the fast beam $n_{f}=\left(n_{p} \times n_{\text {syn }}\right)^{1 / 2}$ to ensure $n_{f} / n_{p} \simeq 0.01$ and $n_{\text {syn }} / n_{f}=0.01$, where $n_{\text {syn }}$ is the proper density of the emitting particles of the fast component. Two different approaches have been made for the bulk velocity: $V_{0}=V_{A}=0.02 c$ where $V_{A}$ is the Alfven velocity in the critical region and a maximal limit, $V_{0}=\frac{V_{A}}{t g \alpha}$ where $\alpha$ is the opening angle of the slow component.

The results give new light on the classification of AGNs. A smaller ratio $K_{f} / K_{0}$ for the WATs than for the FRIs suggests a stronger influence of the fast beam in the FRIs. The energy flux distribution versus the distance to the critical zone shows that WATs extend themselves through a large domain of critical distances $(10-110 \mathrm{kpcs})$, always keeping small values of $K_{f} / K_{0}=3$ (for $V_{0}=V_{A}$ ) while FRIs are grouped near the short critical distances $<10$ kpcs together with the remaining sources studied; however, the domain of $K_{f} / K_{0}\left(5-25\right.$ for $\left.V_{0}=V_{A}\right)$ is much more extended. We found a certain tendency to higher nucleus activity $(L x)$ for higher $K_{f} s$ though we did not find data for all the sources. A larger sample should be studied to further investigate the possibility of a correlation between the large scale inflow $\dot{M}_{\text {cool }}$ and the ejection from the slow component.

T. J.-L. Courvoisier and A. Blecha: Multi-Wavelength Continuum Emission of AGN, 433. (C) 1994 IAU. Printed in the Netherlands. 